Proceedings of the 9th Workshop on Quantum Chaos and Localisation Phenomena, May 24-26, 2019, Warsaw, Poland

\title{
Understanding Quantum Graphs
}

\author{
P. KuRASOV \\ Dept. of Mathematics, Stockholm Univ., 10691 Stockholm, Sweden
}

\begin{abstract}
Current understanding of spectral asymptotics for quantum graphs is described. It is discussed how these results can be applied to inverse problems. In particular it is analysed whether the inverse spectral problem has a unique solution in the spirit of classical Ambartsumian theorem: a complete overview is provided.
\end{abstract}

DOI: 10.12693/APhysPolA.136.797

PACS/topics: quantum graphs, spectral asymptotics, isospectrality

\section{Introduction}

Quantum graphs - ordinary differential equations on metric graphs - have attracted a lot of attention in recent years [1-3]. These models were used by physicists due to their simplicity and rich spectral properties, in particular as an explicit model for transition from regular to chaotic behaviour (see, e.g., $[4-8]$ ). One has even performed experiments using microwave networks to simulate quantum graphs $[9,10]$. Mathematicians were interested in spectral properties of quantum graphs [11-18], in particular, understanding structure of nodal domains $[5,19,20]$ allowing, for example, to characterise trees [21]. Most attractive approaches to spectral theory of graph Laplacians are based on scattering ideas $[7,22,23]$ and on studies of the determinant manifold $[24,25]$. Using the first approach one may prove trace formula $[7,22,23,26-30]$ connecting the spectrum to the set of periodic orbits on the metric graph.

The goal of this note is to describe recent developments in the spectral theory of quantum graphs $[1,2]$ connected with spectral asymptotics and inverse spectral problems. Under the inverse spectral problem we understand here reconstruction of a quantum graph from its spectrum. Our focus will be on results that remind Ambartsumian theorem from 1929 which states that the spectrum uniquely determines the operator in the case of Neumann Laplacian on a compact interval [31]. The Schrödinger operators on metric graphs, known as quantum graphs, possess properties of ordinary and partial differential equations, but their spectral asymptotics are unusual. For example, for non-trivial metric graphs (not a single interval, not a loop), it is impossible to improve Weyl's asymptotics in the sense that no lower term can be added to Weyl's term.

It appears that spectra of quantum graphs can be classified by gathering together isospectral Laplacians and corresponding Schrödinger operators. The spectra of the Laplace and the Schrödinger operators (on the same metric graph and with the same vertex conditions)

corresponding author; e-mail: kurasov@math.su.se are asymptotically close and it appears that they always belong to the same spectral cluster.

Studies of inverse spectral problems for quantum graphs is at its starting point, hence we focus here on different generalisations of the celebrated Ambartsumian theorem (for the Schrödinger operator on a single interval), which was a source of inspiration for decades when the inverse spectral theory in one dimension was developed.

\section{Preliminaries}

\subsection{Definitions}

A quantum graph is a triple consisting of a metric graph $\Gamma$, a differential expression acting on the edges and vertex conditions imposed at the vertices. A metric graph is a finite collection of compact intervals $E_{n}=\left[x_{2 n-1}, x_{2 n}\right], n=1,2, \ldots, N$, with some of the end points identified forming vertices $V_{m}$ (see Fig. 1). In this picture every vertex is an equivalence class of end points from $\boldsymbol{V}=\left\{x_{j}\right\}_{j=1}^{2 N}$. With $\Gamma$ we associate the Hilbert space

$$
L_{2}(\Gamma)=\bigoplus_{n=1}^{N} L_{2}\left(E_{n}\right) .
$$

Note that $L_{2}(\Gamma)$ does not reflect how the edges are connected. We shall reduce our studies to the Schrödinger differential expressions $-\frac{\mathrm{d}^{2}}{\mathrm{~d} x^{2}}+q(x)$ with real summable potential $q \in L_{1}(\Gamma)$. To make such differential expression self-adjoint in $L_{2}(\Gamma)$ one needs to select vertex conditions connecting the limit values of the functions $\boldsymbol{u}_{m}$ and their directed derivatives $\partial \boldsymbol{u}_{m}$ at each vertex $V_{m}$ :

$$
\mathrm{i}\left(S^{m}-I\right) \boldsymbol{u}_{m}=\left(S^{m}+I\right) \partial \boldsymbol{u}_{m},
$$

where $S^{m}$ is any irreducible unitary $d_{m} \times d_{m}$ matrix. Its dimension is determined by the degree $d_{m}$ of the vertex coinciding with the number of end points in the equivalence class [32].

We denote the corresponding self-adjoint operator by $L_{q}^{S}(\Gamma)$, where $S$ is the collection of all unitary matrices $S^{m}$. In the special case of standard vertex conditions

$$
\left\{\begin{array}{l}
u \text { is continuous at } V_{m}, \\
\sum_{x_{j} \in V_{m}} \partial u\left(x_{j}\right)=0 \text { (Kirchhoff's condition), }
\end{array}\right.
$$




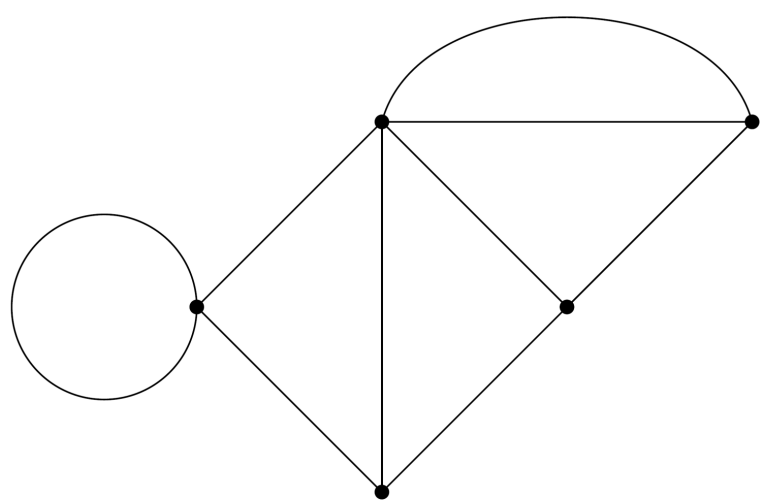

Fig. 1. Connected metric graph.

the operator will be denoted by $L_{q}^{\mathrm{st}}(\Gamma)$. The corresponding Laplacian $L_{0}^{\text {st }}(\Gamma)$ is uniquely determined by the metric graph $\Gamma$.

It is well-known that the spectrum of $L_{q}^{S}(\Gamma)$ is always discrete satisfying Weyl's asymptotics

$$
\lambda_{n}=k_{n}^{2} \sim\left(\frac{\pi}{\mathcal{L}}\right)^{2} n^{2},
$$

where $\mathcal{L}=\sum_{n=1}^{N} \ell_{n}, \ell_{n}=x_{2 n}-x_{2 n-1}$ is the total length of the metric graph $\Gamma$. As we already mentioned no further asymptotic expansion of the form

$$
k_{n}=\frac{\pi}{\mathcal{L}} n+c_{0}+c_{-1} \frac{1}{n}+\ldots
$$

is possible unless $\Gamma$ is an interval or a loop.

\subsection{Two examples}

\subsubsection{Equilateral graphs}

Consider the standard Laplacian on any equilateral metric graph, then the spectrum is periodic [29] (in $k$ ), i.e., there exists $n_{1} \in \mathbb{N}$, such that

$$
k_{n}=\underbrace{\frac{\pi}{\mathcal{L}}\left[\frac{n}{n_{1}}\right] n_{1}+k_{\left\{\frac{n}{n_{1}}\right\}} n_{1}}_{=\frac{\pi}{\mathcal{L}} n+O(1)} .
$$

The spectrum is uniformly discrete but multiple eigenvalues may occur.

\subsubsection{Lasso graph}

The spectrum of the standard Laplacian on the lasso graph formed by two edges of rationally independent lengths $2 \ell_{1}$ and $\ell_{2}$ is given by two series of eigenvalues

- $\frac{\pi}{\ell_{1}} n, \quad n=1,2, \ldots$

- solutions to the equation

$$
3 \sin k\left(\ell_{1}+\ell_{2}\right)+\sin k\left(\ell_{1}-\ell_{2}\right)=0 .
$$

The spectrum is discrete but not uniformly discrete since arbitrarily close eigenvalues occur. We would like to understand how two such different types of spectral behaviour can be unified. In physics language we may speak about regular and chaotic systems.

\section{Inverse problems and exceptional parameters}

To solve the inverse problem one has to reconstruct all three members of the quantum graph triple

- the metric graph $\Gamma$;

- the real potential $q(x) \in L_{1}(\Gamma)$;

- the vertex conditions, i.e., the matrices $S^{m}$.

Even in the case of single interval one spectrum in general is not enough to reconstruct the potential (with the exception of the Ambartsumian theorem). Hence we shall need to consider exceptional parameters in the triple:

- Single interval $[0, \ell]$ as a metric graph - this graph has the smallest Laplacian spectral gap among all graphs of the same total length.

- Zero potential $q(x) \equiv 0$ - the only potential that can be determined a priori without knowing the metric graph.

- Standard vertex conditions - these conditions appear if one requires just continuity of the functions from the quadratic form domain.

In what follows we shall try to understand whether it is possible to prove an analog of the Ambartsumian theorem in the case where some of the parameters in the triple determining quantum graph are assumed to coincide with the exceptional ones and the other parameters are allowed to vary.

\section{Two parameters are fixed}

\subsection{Single interval with Neumann conditions - Ambartsumian theorem}

The following theorem was proven in 1929 by then 21 year-old Victor Ambartsumian*

Theorem 1. Let the spectrum of the one-dimensional Schrödinger operator $-\frac{\mathrm{d}^{2}}{\mathrm{~d} x^{2}}+q(x), q(x) \in \mathbb{R}$ on the interval $[0, \ell]$ with Neumann conditions at the end points coincide with the spectrum of the Neumann Laplacian on the same interval, then the potential is equal to zero (almost everywhere), or symbolically for $n=1,2, \ldots$

$$
\begin{aligned}
& \lambda_{n}\left(L_{q}^{\mathrm{st}}([0, \ell])\right)=\lambda_{n}\left(L_{0}^{\mathrm{st}}([0, \ell])\right) \Leftrightarrow q(x) \equiv 0, \\
& \left.\lambda_{n}\left(L_{q}^{\mathrm{st}}([0, \ell])\right)=\left(\frac{\pi}{\ell}\right)^{2}(n-1)^{2}\right) .
\end{aligned}
$$

Proof. Modern proof of this theorem [33] is based on first deriving the spectral asymptotics using transformation operator

\footnotetext{
* It surprised me that motivation for these studies is pure quantum mechanical despite it was just 1929. Try to read this old paper - it is worth doing!
} 
$k_{n}\left(L_{q}^{\mathrm{st}}([0, \ell])\right)=\frac{\pi}{\ell}\left(n+\frac{\int_{0}^{\ell} q(x) \mathrm{d} x}{\ell} \frac{1}{2}\left(\frac{\ell}{\pi}\right)^{2} \frac{1}{n}+o(1 / n)\right)$,

which implies that

$$
\int_{0}^{\ell} q(x) \mathrm{d} x=0
$$

since $k_{n}\left(L_{q}^{\mathrm{st}}([0, \ell])\right)=\frac{\pi}{\ell} n$ by the assumption of the theorem. Then plugging in $u(x) \equiv 1$ as a trial function into the quadratic form

$$
\int_{0}^{\ell}\left|\psi^{\prime}(x)\right| \mathrm{d} x+\int_{0}^{\ell} q(x)|\psi(x)|^{2} \mathrm{~d} x=0,
$$

we see that it is a minimiser and hence is the ground state

$$
-\psi^{\prime \prime}(x)+q(x) \psi(x)=0 \Rightarrow q(x) \equiv 0 .
$$

\subsection{Standard Laplacian on arbitrary metric graph - geometric version of Ambartsumian theorem}

Theorem 2. Assume that the spectrum of the standard Laplacian on a metric graph $\Gamma$ coincides with the spectrum of the Neumann Laplacian on the interval, then $\Gamma$ (essentially) coincides with the interval, or symbolically

$$
\lambda_{n}\left(L_{0}^{\mathrm{st}}(\Gamma)\right)=\lambda_{n}\left(L_{0}^{\mathrm{st}}([0, \ell])\right) \Leftrightarrow \Gamma=[0, \ell] .
$$

Proof. From Weyl's law we see that the metric graph and the interval should have the same total length. Moreover, the spectral gaps for the two Laplacians coincide. Nicaise [34], Friedlander [18], and Naboko [35] (see also [15]) proved by different methods that interval has the smallest spectral gap among all graphs of the same length. Hence, $\Gamma$ is the interval since it has the smallest possible spectral gap. One should only be careful and remove all dummy vertices of degree two, since standard conditions at such vertices are equivalent to continuity of the function and its first derivative.

\subsection{Laplacian on the interval}

We add this case for the sake of completeness. Let $L_{0}^{\boldsymbol{h}}([0, \ell])$ denote the Laplacian on the interval $[0, \ell]$ with the Robin conditions at the end points

$$
\boldsymbol{h}=\left(h_{0}, h_{1}\right) \in \mathbb{R}^{2} \Rightarrow\left\{\begin{array}{l}
\partial u(0)=h_{0} u(0), \\
\partial u(\ell)=h_{1} u(\ell) .
\end{array}\right.
$$

Theorem 3. Two Laplacians on an interval $[0, \ell]$ with the Robin conditions at the end points are isospectral if and only if the Robin parameters are equal up to permutation

$$
\begin{aligned}
& \lambda_{n}\left(L_{0}^{\boldsymbol{h}}([0, \ell])\right)= \\
& \lambda_{n}\left(L _ { 0 } ^ { \boldsymbol { h } ^ { \prime } } ( [ 0 , \ell ) ) \Leftrightarrow \left[\begin{array}{l}
h_{0}=h_{0}^{\prime} \text { and } h_{1}=h_{1}^{\prime}, \\
h_{0}=h_{1}^{\prime} \text { and } h_{1}=h_{0}^{\prime} .
\end{array}\right.\right.
\end{aligned}
$$

Proof. The secular equation for the Robin Laplacian

$$
\left(k-\frac{h_{0} h_{1}}{k}\right) \sin (k \ell)=\left(h_{0}+h_{1}\right) \cos (k \ell)
$$

depends on the sum $h_{0}+h_{1}$ and product $h_{0} h_{1}$ of the parameters, hence the parameters are determined up to the exchange of the end points.

These calculations show that the parameters are determined by the spectrum in this special case, not only if the boundary conditions are Neumann.

\section{One parameter is fixed}

\subsection{Standard Schrödinger operators}

In this section we assume only that the vertex conditions are standard, but the graph and potential are arbitrary.

Theorem 4. If the spectrum of the standard Schrödinger operator on a metric graph $\Gamma$ coincides with the spectrum of the Neumann Laplacian on an interval, then the graph (essentially) coincides with the interval and the potential is zero, or symbolically

$$
\lambda_{n}\left(L_{q}^{\mathrm{st}}(\Gamma)\right)=\lambda_{n}\left(L _ { 0 } ^ { \mathrm { st } } ( [ 0 , \ell ] ) \Leftrightarrow \left\{\begin{array}{l}
\Gamma=[0, \ell], \\
q(x) \equiv 0 .
\end{array}\right.\right.
$$

Note that this theorem is not a simple combination of the classical Ambartsumian Theorem 1 and its geometric version (Theorem 2).

Proof. The theorem can be proven in four steps (see [36]):

1. The spectra of the Schrödinger $L_{q}^{\text {st }}(\Gamma)$ and Laplace $L_{0}^{\mathrm{st}}(\Gamma)$ operators on the same graph with the same vertex conditions are asymptotically close:

$$
k_{n}\left(L_{q}^{\mathrm{st}}(\Gamma)\right)-k_{n}\left(L_{0}^{\mathrm{st}}(\Gamma)\right)=o(1) .
$$

Here it is important to take into account that the eigenvalues satisfy Weyl's asymptotic. The proof is elementary if $q \in L_{\infty}(\Gamma)$, but is extended for $q \in$ $L_{1}(\Gamma)$ in [36].

2. The spectrum of the Laplacian is given by a trigonometric polynomial and therefore is close to integers, provided $\ell=\pi$, if and only if it coincides with the integers. It follows that the spectrum of the Laplacian on $\Gamma$ coincides with the spectrum of the Neumann Laplacian on the interval.

3. Geometric version of the Ambartsumian theorem (Theorem 2) implies that the graph $\Gamma$ is essentially the interval.

4. Classical Ambartsumian Theorem 1 implies that $q(x) \equiv 0$. 


\subsection{Schrödinger operators}

with Robin conditions on the interval

In this section the graph is fixed to coincide with the interval. We should analyse whether the spectrum of the Schrödinger operator with the Robin conditions determine the operator uniquely. It is well-known that to reconstruct potential one needs to know two spectra corresponding to different boundary conditions [33, 37-39].

We present here an explicit counterexample using Crum's article [40], where Darboux transform was applied to add eigenvalues to the Schrödinger operator on the interval.

Start with the Dirichlet Laplacian: $\lambda_{n}=\left(\frac{\pi n}{\ell}\right)^{2}$, $n=1,2, \ldots$ Its spectrum differs from the Neumann Laplacian by just one eigenvalue $\lambda=0$. We add this eigenvalue by Crum's method. If we scale the interval to $[0,1]$, then we get the potential and Robin parameters equal to

$$
q(x)=\frac{-1}{x+1}, \quad h_{0}=-1, \quad h_{1}=\frac{1}{2} .
$$

Moreover, the corresponding eigenfunctions are explicit $\psi_{1}(x)=\frac{1}{x+1}, \quad \psi_{n+1}=-\frac{1}{\pi^{2} n^{2}}\left(n \cos (n x)-\frac{\sin (n x)}{x+1}\right)$.

We constructed a family of Robin Schrödinger operators isospectral to the Neumann Laplacian, hence no Ambartsumian-type theorem holds in this case.

\subsection{Laplace operators on arbitrary graphs with arbitrary vertex conditions}

Here we again present an explicit counterexample implying that no Ambartsumian-type theorem is possible. Let $\Gamma$ be the graph formed by the two intervals of length $1 / 2$ connected at one vertex. We assume standard (= Neumann) conditions at the outer vertices. Conditions at the central vertex are given as

$$
\mathrm{i}(S-I)\left(\begin{array}{l}
u\left(x_{1}\right) \\
u\left(x_{2}\right)
\end{array}\right)=(S+I)\left(\begin{array}{l}
\partial u\left(x_{1}\right) \\
\partial u\left(x_{2}\right)
\end{array}\right)
$$

with the $2 \times 2$ matrix $S$ unitary and Hermitian

$$
S^{-1}=S^{*}=S \text {. }
$$

Then the following holds:

$$
\lambda_{n}\left(L_{0}^{S, \text { st }}(\Gamma)\right)=\lambda_{n}\left(L_{0}^{\mathrm{st}}(I)\right) .
$$

The proof is essentially based on the fact that $S^{2}=\mathbb{I}$ and explicit calculation of the ground state. Every such $2 \times 2$ matrix possesses the representation

$$
S(a, \theta)=\left(\begin{array}{cc}
a & \sqrt{1-a^{2}} \mathrm{e}^{\mathrm{i} \theta} \\
\sqrt{1-a^{2}} \mathrm{e}^{-\mathrm{i} \theta} & -a
\end{array}\right) .
$$

This family interpolates between the case of single interval (standard vertex conditions at the central vertex) and two intervals with the Dirichlet and Neumann conditions

$$
\underbrace{\left(\begin{array}{ll}
0 & 1 \\
1 & 0
\end{array}\right)}_{a=0} \leftrightarrow \underbrace{\left(\begin{array}{cc}
1 & 0 \\
0 & -1
\end{array}\right)}_{a=1} .
$$

\section{Further extensions}

Counterexamples presented in the last two subsections imply that no general Ambartsumian-type theorem, where all three parameters may vary is possible. Therefore, we discuss here two more extensions close to the Ambartsumian theorem in their spirit.

\subsection{A theorem by Brian Davies}

Theorem [41] Let arbitrary finite compact metric graph $\Gamma$ be fixed, then the standard Schrödinger operator is isospectral to the standard Laplacian if and only if the potential is zero, or symbolically

$$
\lambda_{n}\left(L_{q}^{\mathrm{st}}(\Gamma)\right)=\lambda_{n}\left(L_{0}^{\mathrm{st}}(\Gamma)\right) \Rightarrow q(x) \equiv 0 .
$$

Proof. To prove the theorem it is enough to show that

$$
\int_{\Gamma} q(x) \mathrm{d} x=0
$$

then the proof of classical Ambartsumian Theorem 1 can just be repeated step by step, since we know that the ground state is zero $\lambda_{1}\left(L_{q}^{\mathrm{st}}(\Gamma)\right)=\lambda_{1}\left(L_{0}^{\mathrm{st}}(\Gamma)\right)=0$.

Let $H_{\Gamma}$ be the heat kernel for $L_{0}^{\text {st }}(\Gamma)$ :

$$
\lim _{t \rightarrow 0} \sqrt{t} H_{\Gamma}(t, x, x)=\frac{1}{\sqrt{4 \pi}}, \quad x \in \Gamma \backslash\left(\cup_{m=1}^{M} V_{m}\right) .
$$

Perturbation formula for traces of the heat semigroups implies that

$$
\begin{gathered}
\operatorname{Tr}\left(\mathrm{e}^{-L_{q}^{\mathrm{st}}(\Gamma) t}\right)-\operatorname{Tr}\left(\mathrm{e}^{-L_{0}^{\mathrm{st}}(\Gamma) t}\right)= \\
-t \int_{\Gamma} H_{\Gamma}(t, x, x) q(x) \mathrm{d} x+\rho(t),
\end{gathered}
$$

where $\rho(t)=O\left(t^{3 / 2}\right)$. The left hand side is identically zero, hence taking the limit $t \rightarrow 0$ we get the desired equality (11).

The theorem is proven for $q \in L_{\infty}(\Gamma)$ in [41], but it is indicated that it should hold also for $q \in L_{1}(\Gamma)$.

\subsection{A theorem by P.K. and Rune Suhr}

Using that the spectra of standard Laplacians are described by trigonometric polynomials, which are almost periodic holomorphic functions one may prove the following theorem [42, 43].

Theorem 6. Assume that the Laplacians on $\Gamma_{1}$ and $\Gamma_{2}$ are asymptotically isospectral

$$
k_{n}\left(L_{0}^{\mathrm{st}}\left(\Gamma_{1}\right)\right)-k_{n}\left(L_{0}^{\mathrm{st}}\left(\Gamma_{2}\right)\right) \rightarrow 0
$$

then the operators are isospectral.

It is important to bear in mind that there exists isospectral Laplacian in the case of rationally dependent edge lengths [22]. In fact, this theorem can be generalised to include arbitrary almost periodic functions, and not only trigonometric polynomials. It implies that zeroes of almost periodic functions have certain rigidity - they cannot be perturbed a little. In applications all measurements lead to eigenvalues known with certain precision. Using rigidity of the zeroes one may improve the measured values. 


\subsection{Implications of the two theorems}

The two above cited theorems lead to the following interesting properties concerning spectra of quantum graphs.

Theorem 7. Two Schrödinger operators $L_{q_{1}}^{\mathrm{st}}\left(\Gamma_{1}\right)$ and $L_{q_{2}}^{\mathrm{st}}\left(\Gamma_{2}\right)$, on a maybe different metric graphs $\Gamma_{1}$ and $\Gamma_{2}$ respectively, are asymptotically isospectral if and only if the Laplacians

$$
L_{0}^{\mathrm{st}}\left(\Gamma_{1}\right) \text { and } L_{0}^{\mathrm{st}}\left(\Gamma_{2}\right)
$$

are isospectral, or symbolically

$$
\begin{gathered}
k_{n}\left(L_{q_{1}}^{\mathrm{st}}\left(\Gamma_{1}\right)\right)-k_{n}\left(L_{q_{2}}^{\mathrm{st}}\left(\Gamma_{2}\right)\right)=o(1) \Leftrightarrow \\
k_{n}\left(L_{0}^{\mathrm{st}}\left(\Gamma_{1}\right)\right)=k_{n}\left(L_{0}^{\mathrm{st}}\left(\Gamma_{2}\right)\right) .
\end{gathered}
$$

Proof. Using (8) we see that the Schrödinger and Laplace operators on the same metric graph with standard vertex conditions are asymptotically isospectral (see (12)). Hence the Laplacians are asymptotically isospectral as well, then Theorem 6 implies that the Laplacians are isospectral.

Assume now that the Schrödinger operator on one metric graph is isospectral to the Laplacian on a maybe different metric graph. Again we assume standard vertex conditions.

Theorem 8. Let $\Gamma_{1,2}$ be two metric graphs, then the Schrödinger operator $L_{q}^{\text {st }}\left(\Gamma_{1}\right)$ is isospectral to the Laplacian $L_{0}^{\text {st }}\left(\Gamma_{2}\right)$ only if

$$
q(x) \equiv 0 .
$$

Proof. The Laplacian $L_{0}^{\mathrm{st}}\left(\Gamma_{1}\right)$ is asymptotically isospectral to $L_{0}^{\mathrm{st}}\left(\Gamma_{2}\right)$, hence by Theorem 6 they are isospectral. It follows that the Schrödinger and Laplace operators on $\Gamma_{1}$ are isospectral, then Theorem 5 by Davies implies $q(x) \equiv 0$.

\section{Conclusion}

- The spectra of standard Laplacians possess certain rigidity: even under asymptotically small perturbations the spectra fit into certain equivalence classes.

- Spectral asymptotics do not determine the metric graph, but they do determine the isospectrality class of graphs.

- Two different types of spectral behaviour (equilateral graphs compared to graphs with rationally independent edge lengths) are explained as different zero types of almost periodic functions.

\section{References}

[1] G. Berkolaiko, P. Kuchment, Introduction to Quantum Graphs, Mathematical Surveys and Monographs, Vol. 186, American Mathematical Society, Providence (RI) 2013.

[2] P. Kurasov, Quantum Graphs: Spectral Theory and Inverse Problems, to appear.
[3] O. Post, "Spectral analysis on graph-like spaces", in: Lecture Notes in Mathematics, Vol. 2039, Springer, Heidelberg 2012.

[4] L. Alon, R. Band, G. Berkolaiko, Commun. Math. Phys. 362, 909 (2018)

[5] S. Gnutzmann, J.P. Keating, F. Piotet, Ann. Phys. 325, 2595 (2010).

[6] S. Gnutzmann, A. Altland, Phys. Rev. E 72, 056215 (2005).

[7] T. Kottos, U. Smilansky, Ann. Phys. 274, 76 (1999).

[8] T. Kottos, U. Smilansky, J. Phys. A 36, 3501 (2003).

[9] M. Lawniczak, A. Sawicki, S. Bauch, M. Kuś, L. Sirko, Phys. Rev. E 89, 032911 (2014).

[10] M. Lawniczak, J. Lipovský, L. Sirko, Phys. Rev. Lett. 122, 140503 (2019).

[11] R. Band, G. Lévy, Ann. Henri Poincaré 18, 3269 (2017).

[12] R. Band, O. Parzanchevski, G. Ben-Shach, J. Phys. A 42, 175202 (2009).

[13] R. Band, A. Sawicki, U. Smilansky, J. Phys. A 43, 415201 (2010).

[14] G. Berkolaiko, J.B. Kennedy, P. Kurasov, D. Mugnolo, J. Phys. A 50, 365201 (2017).

[15] G. Berkolaiko, J.B. Kennedy, P. Kurasov, D. Mugnolo, Trans. Am. Math. Soc. 372, 5153 (2019).

[16] G. Berkolaiko, Y. Latushkin, S. Sukhtaiev, Adv. Math. 352, 632 (2019).

[17] G. Berkolaiko, W. Liu, J. Math. Anal. Appl. 445, 803 (2017).

[18] L. Friedlander, Ann. Inst. Fourier (Grenoble) 55, 199 (2005).

[19] R. Band, G. Berkolaiko, H. Raz, U. Smilansky, Commun. Math. Phys. 311, 815 (2012).

[20] G. Berkolaiko, B. Winn, Nonlinearity 31, 4812 (2018).

[21] R. Band, Philos. Trans. R. Soc. Lond. Ser. A 372, 20120504 (2014).

[22] B. Gutkin, U. Smilansky, J. Phys. A 34, 6061 (2001).

[23] P. Kurasov, M. Nowaczyk, J. Phys. A 38, 4901 (2005).

[24] F. Barra, P. Gaspard, J. Statist. Phys. 101, 283 (2000).

[25] Y.C. de Verdière, Ann. Henri Poincaré 16, 347 (2015).

[26] J. Bolte, S. Endres, in: Proc. Symp. Pure Math., Vol. 77, Amer. Math. Soc., Providence (RI) 2008, p. 247.

[27] J. Bolte, S. Endres, Ann. Henri Poincaré 10, 189 (2009).

[28] P. Kurasov, Ark. Mat. 46, 95 (2008).

[29] P. Kurasov, J. Funct. Anal. 254, 934 (2008).

[30] R. Rueckriemen, U. Smilansky, J. Phys. A 45, 475205 (2012).

[31] V. Ambartsumian, Z. Phys. 53, 690 (1929).

[32] P. Kurasov, M. Nowaczyk, Opuscula Math. 30, 295 (2010).

[33] G. Borg, in: C.R. Dixième Congrès Math. Scandinaves, 1946, Jul. Gjellerups Forlag, Copenhagen 1947, p. 172. 
[34] S. Nicaise, Bull. Sci. Math. 111, 401 (1987) (in French).

[35] P. Kurasov, S. Naboko, J. Spectr. Theory 4, 211 (2014).

[36] J. Boman, P. Kurasov, R. Suhr, Integr. Equ. Oper. Theory 90, 40 (2018).

[37] G. Borg, Acta Math. 78, 1 (1946) (in German).

[38] I.M. Gel'fand, B.M. Levitan, Izv. Akad. Nauk SSSR. Ser. Mat. 15, 309 (1951) (in Russian).

[39] B.M. Levitan, M.G. Gasymov, Mat. Sb. (N.S.) 63 (105), 445 (1964) (in Russian).
[40] M.M. Crum, Quart. J. Math. Oxford Ser. 2 6, 121 (1955).

[41] E.B. Davies, J. Oper. Theory 69, 195 (2013).

[42] P. Kurasov, R. Suhr, J. Math. Phys. 59, 102104 (2018).

[43] P. Kurasov, R. Suhr, "Asymptotically isospectral quantum graphs and generalised trigonometric polynomials", Research Reports in Mathematics Number 2, 2017, Dept. of Mathematics, Stockholm Univ., submitted for publication. 